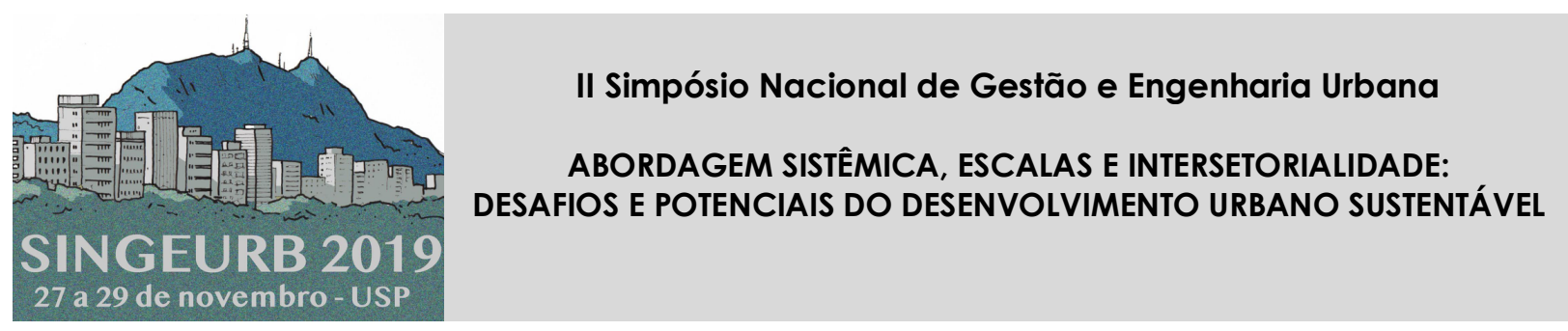

\title{
Transporte ativo em um campus universitário urbano: caso da UFMT ${ }^{1}$
}

\section{Active transport on an urban university campus: the case of UFMT}

\section{Medeiros, Matheus Guedes'; Lopes, João Paulo Barbosa2; Bender, Juliane Érika Cavalcante 3 ; Baltar, Marina Leite de Barros ${ }^{4}$}

\author{
1 Universidade Federal de Mato Grosso, Av. Fernando Corrêa da Costa, $n^{\circ}$ \\ 2367, Bairro Boa Esperança. Brasil.matheus_g.medeiros@hotmail.com \\ 2 Universidade Federal de Mato Grosso, joaopauloblopes@gmail.com \\ 3 Universidade Federal de Mato Grosso, bender@pet.coppe.ufrj.br \\ ${ }^{4}$ Universidade Federal de Mato Grosso, mabaltar@gmail.com
}

\begin{abstract}
RESUMO
Instituições de ensino são consideradas Polos Geradores de Viagens (PGV) em razão da quantidade e características das viagens de alunos, professores, técnicos e visitantes, que acabam por acarretar problemas de mobilidade. O presente estudo se propõe a traçar o perfil dos usuários que utilizam o transporte ativo para acessar o campus da Universidade Federal de Mato Grosso (UFMT) em Cuiabá. Foram utilizados os dados provenientes de uma pesquisa O/D on-line realizada nos meses de fevereiro e março de 2019, sendo esta vinculada a um projeto de pesquisa focado na promoção da mobilidade sustentável no campus. Após a análise dos resultados percebeu-se que as mulheres se sentem mais inseguras ao caminhar ou pedalar para o campus. Além disso, os deslocamentos cobrem, em média, 1,51 km e têm duração de aproximadamente 18 minutos para os pedestres e 6 minutos para os ciclistas. Os picos de entrada e saída foram respectivamente, das 7 h00 às 08 h00 e das 17 h00 às $18 \mathrm{h00}$. As reclamações mais recorrentes foram em relação à falta de segurança, infraestrutura de transporte precária e ao calor excessivo de Cuiabá.
\end{abstract}

Palavras-chave: transporte ativo, campus universitário, pesquisa origem-destino.

\begin{abstract}
Educational institutions can be understood as trip generating developments considering the quantity and characteristics of the trips of students, teachers and technicians. The present study presents the profile of active transportation users that access the campus of Universidade Federal de Mato Grosso (UFMT) in Cuiabá. An online survey was conducted during the months of February and March in 2019 and it was used as the data source for this study. The survey is linked to a research project focused on promoting sustainable mobility on campus. The results show that women feel more insecure while walking or cycling. Furthermore, the trips cover a medium distance of 1,51 kilometers, lasting 18 minutes when walking, and six minutes by bicycle. The morning-peak begins at 7 am and finishes at $8 \mathrm{am}$, whilst the afternoon-peak starts
\end{abstract}

\footnotetext{
1 MEDEIROS, Matheus Guedes; LOPES, João Paulo Barbosa; BENDER, Juliane Érika Cavalcante; BALTAR, Marina Leite de Barros. Transporte ativo em um campus universitário urbano: caso da UFMT. In: II SIMPÓSIO NACIONAL DE GESTÃo E ENGENHARIA URBANA: SINGEURB, 2019, São Paulo. Anais... Porto Alegre: ANTAC, 2019.
} 
at $5 \mathrm{pm}$ ending at $6 \mathrm{pm}$. The most recurrent complaints were regarding the lack of security, poor active transportation infrastructure and Cuiaba's excessive heat.

Key-words: Active transportation, college campus, origin-destination survey.

\section{INTRODUÇÃO}

Polos Geradores de Viagens (PGVs) são empreendimentos de grande porte que atraem ou produzem grande número de viagens (DENATRAN, 2001). Durante seu processo de implantação e planejamento devem ser considerados os potenciais impactos no sistema viário e de transporte, na estrutura urbana, no desenvolvimento socioeconômico e na qualidade de vida da população (KNEIB, 2004).

O estudo é focado na Universidade Federal de Mato Grosso (UFMT), campus Cuiabá, compreendida na categoria de estabelecimentos de ensino, de acordo com Portugal e Goldner (2003). Para Stein, Rodrigues da Silva e Júnior (2012), o interesse de pesquisadores em estudar a mobilidade em instituições de ensino surge em razão da quantidade e características das viagens de alunos, professores, técnicos e visitantes, que acabam por acarretar problemas de mobilidade semelhantes à de outros PGVs. Apesar disso, o ambiente da universidade favorece o desenvolvimento e implantação de modelos de gerenciamento da demanda que, se eficientes, podem ser posteriormente transferidos à sociedade (PARRA, 2006).

Este texto busca caracterizar as viagens dos usuários que utilizam o transporte ativo para acessar a UFMT, analisando sua idade, gênero, renda, tempo despendido e horário de entrada e saída do campus. Tem-se como objetivo compreender os deslocamentos desta modalidade de transporte - através das distâncias de viagem, duração e origem. Pretendese utilizar este diagnóstico para em trabalhos futuros propor soluções e medidas para incentivar o uso desta alternativa de transporte na instituição.

\section{REVISÃO DA LITERATURA}

Há um pouco mais de 20 anos, através do estudo de Tolley (1996), observa-se o surgimento do interesse pela promoção da mobilidade sustentável em campi universitários. O autor apresenta uma discussão acerca das altas taxas de motorização da comunidade acadêmica e o fornecimento de estacionamento gratuito dentro dos campi. Balsas (2003), por sua vez, discute as políticas de incentivo do transporte ativo dentro de oito instituições de ensino superior nos Estados Unidos, elencando as medidas tomadas para o estímulo desse tipo de deslocamento.

Stasko, Buck e Gao (2013) trazem os resultados de uma pesquisa voltada à compreensão dos impactos da implantação de um serviço de carsharing em uma universidade. Riggs (2014), utilizando dados da UC Berkeley, conclui que a diminuição da oferta de estacionamento tem o potencial de aumentar a acessibilidade do campus.

Mikiki e Papadopoulou (2017) argumentam que estudantes podem se tornar criadores de tendências, originando um efeito multiplicador com suas escolhas de viagem. No estudo desenvolvido pelos autores destaca-se a grande aceitação da bicicleta entre os universitários e a necessidade, destacada pelos entrevistados, da ampliação da rede cicloviária da instituição.

Na realidade brasileira podem ser encontrados alguns estudos analisando a mobilidade em campi universitários, como: UFRJ - campus llha do Fundão (CARVALHO, 2016), USP - campus São Carlos (STEIN, 2013) e UFPE - campus Caruaru (ANDRADE et al., 2011). No caso da UFMT campus Cuiabá, não foram encontrados trabalhos focados na compreensão dos deslocamentos gerados pela instituição. 


\section{METODOLOGIA}

A UFMT ocupa uma área com cerca de $739.000 \mathrm{~m}^{2}$, localizada na Avenida Fernando Correa da Costa - Bairro Boa Esperança, possui dois portões de acesso para automóveis e três para uso exclusivo de pedestres. De acordo com o anuário estatístico de 2018 da instituição, a população do campus é de 13.645 pessoas - considerando servidores e discentes (UFMT, 2018).

Utilizou-se um questionário on-line, semelhante ao criado por Carvalho (2016), divulgado via e-mail institucional e mídias sociais ao longo dos meses de fevereiro e março de 2019. Nesse questionário foi realizada uma pesquisa Origem-Destino (O/D), um levantamento do perfil socioeconômico do respondente e do índice de satisfação deste em relação ao modo de transporte utilizado em seus deslocamentos para instituição.

O controle amostral realizado consistiu na verificação dos resultados incompletos ou errados, por falta ou inconsistência da informação ou por se tratar de outros campi da UFMT em cidades diferentes, resultando em uma redução de $2 \%$ da amostra. Dessa forma, restaram-se 1.367 respostas úteis referentes aos deslocamentos de docentes, técnicos, estudantes e usuários externos. Esta amostra corresponde a aproximadamente 12\% da população, com um nível de confiança de $95 \%$ e erro amostral de $3 \%$.

De forma a caracterizar esses usuários foram analisados: idade, gênero, renda, distância média percorrida, horário de entrada e saída e suas avaliações em relação à segurança, conforto e rapidez. Além disso, uma vez que a pesquisa contava com um campo aberto onde os entrevistados eram encorajados a expor suas opiniões e sentimentos em relação ao modo de transporte que utilizavam em seus deslocamentos, este também foi incluído no processo de análise.

Para a definição da distância e do tempo de viagem foi adotado o centróide dos bairros de residência até o Restaurante Universitário (RU), que se localiza na parte central da UFMT. Utilizou-se o Google Maps para traçar o caminho e computar as distâncias. O cálculo do tempo de deslocamento foi realizado assumindo-se uma velocidade média de $5 \mathrm{~km} / \mathrm{h}$ para os pedestres e de $15 \mathrm{~km} / \mathrm{h}$ para os ciclistas, em consonância com os estudos de Segadilha (2014) e Monteiro e Campos (2011).

\section{RESULTADOS E DISCUSSÕES}

Na UFMT, a maioria dos deslocamentos é realizada por transporte individual, somando aproximadamente $47 \%$ do total, seguido pelo transporte público, com $39 \%$, e os demais por transporte ativo. Entre as pessoas entrevistadas que utilizam modos não motorizados: 257 andam a pé e 24 de bicicleta.

Em relação ao gênero, $51 \%$ se identificam com o gênero feminino e $49 \%$ com o masculino, indicando que não há tanta disparidade entre os entrevistados. Quanto à idade, constata-se que quanto mais jovem, maior a tendência a caminhar ou pedalar até a universidade - cerca de $80 \%$ da amostra é composta de usuários entre 17 e 26 anos.

Analisando a renda, é verificado que os usuários do transporte ativo são em sua maioria alunos de graduação e têm renda concentrada, principalmente, nas faixas até $R \$ 3.000$, conforme demonstra a Figura 1. Para $\circ$ grupo dos funcionários da instituição percebe-se rendas superiores, estando majoritariamente entre $R \$ 5.000$ a $R \$ 10.000$. 
Figura 1 - Renda, em $R \$$, dos usuários de transporte ativo

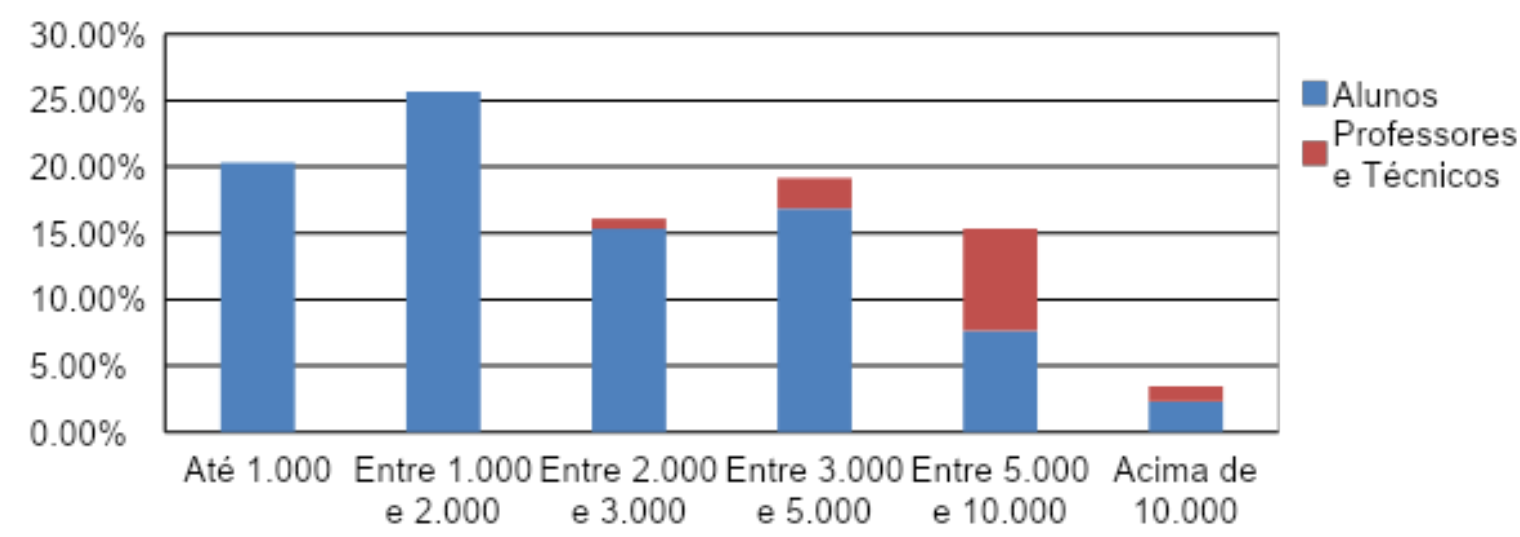

Fonte: Os autores

A Figura 2 apresenta as distâncias percorridas pelos usuários do transporte ativo até a UFMT, considerando o centróide do bairro de origem e o RU. Os resultados obtidos demonstram que a maioria dos que se deslocam a pé ou de bicicleta, cerca de $80 \%$, residem em bairros distantes até 2 quilômetros da instituição. Estima-se que a distância média, calculada através de uma média ponderada, é de $1,51 \mathrm{~km}$, sendo esta uma extensão de viagem aceitável. Analogamente, os tempos de deslocamento são de, aproximadamente, 18 minutos para os pedestres e 6 minutos para ciclistas.

Figura 2 - Usuários por distância entre o centróide do bairro e o restaurante universitário (km)

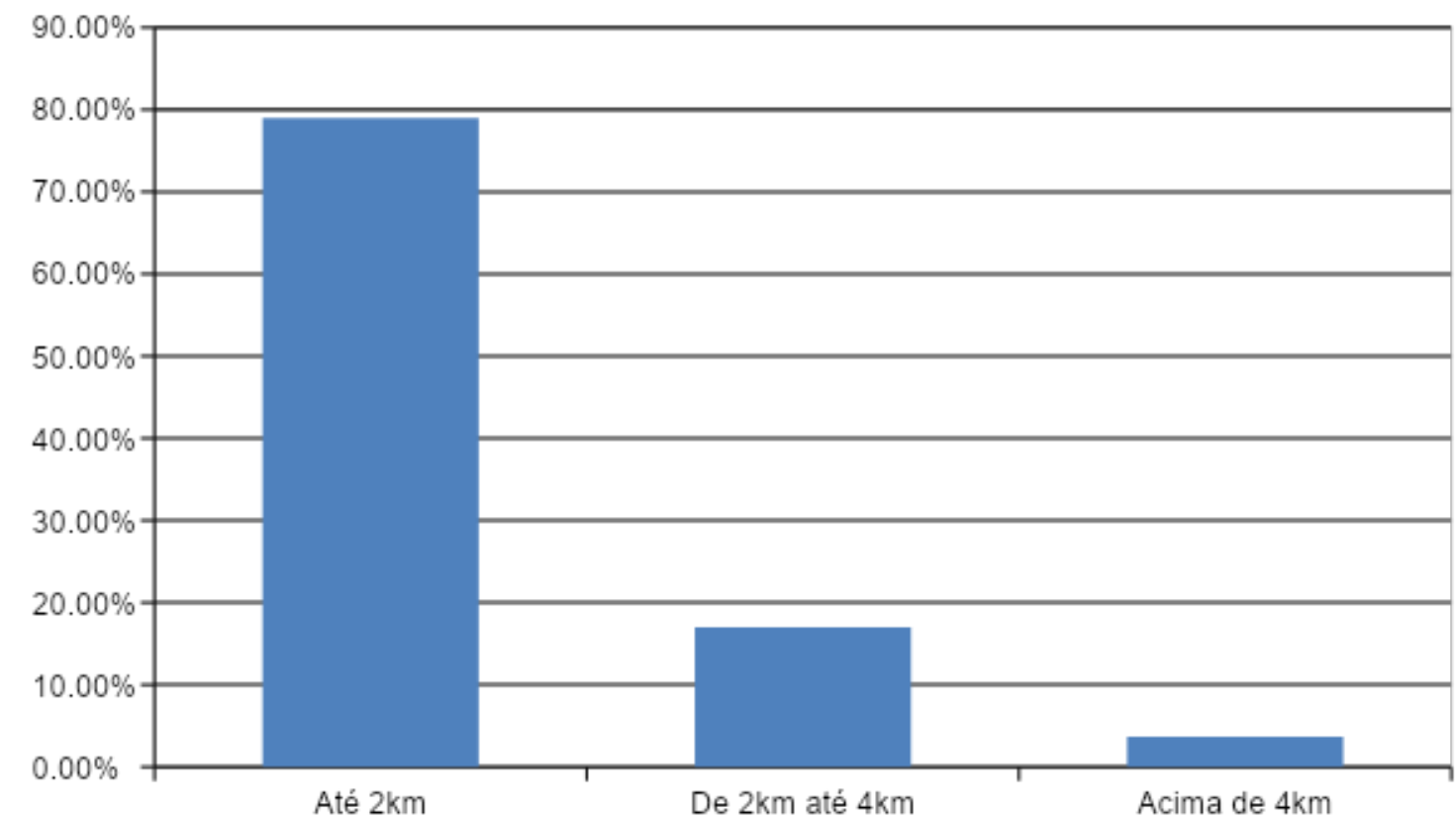

Fonte: Os autores

As respostas sobre o horário de entrada e saída indicam picos entre 07h00 e 08h00 para a chegada à instituição, e das 17 h00 às $18 \mathrm{~h} 00$ para a saída - conforme demonstra a Figura 3. De fato, o resultado era esperado uma vez que a UFMT oferece cursos matutinos, vespertinos e noturnos. É demonstrado, também, que existe a tendência de permanência ao longo de todo o dia na universidade. 
Figura 3 - Picos de chegada e saída do campus

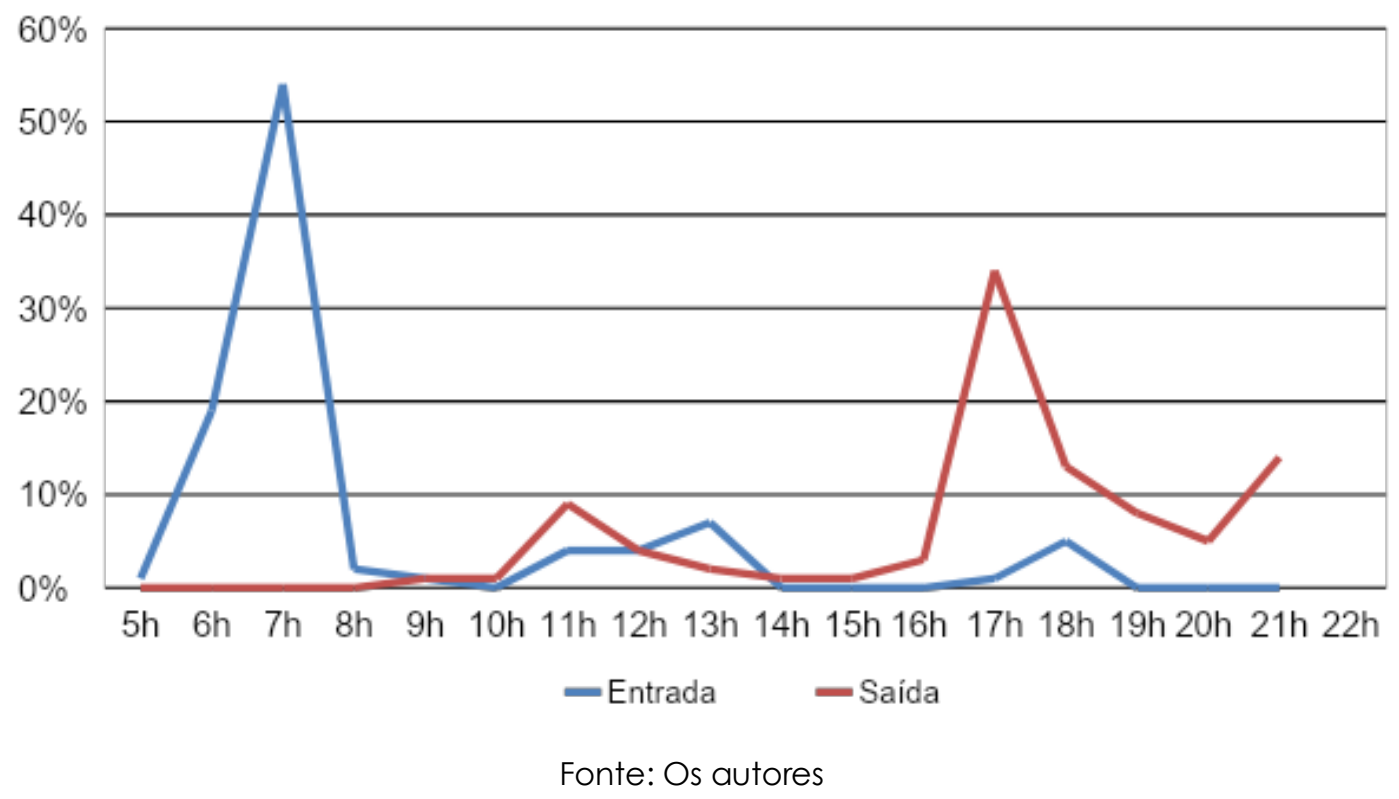

Explorando o campo da pesquisa onde era possível deixar uma opinião ou relatar seus sentimentos em relação ao modo que utilizava em seus deslocamentos, identifica-se a existência de dois principais grupos de pessoas que se deslocam para a UFMT por modos não motorizados. O primeiro grupo faz essa opção por não ter um carro e/ou não estar satisfeito com o transporte público ofertado; o segundo possui outras formas de deslocamento, mas escolhe o ciclismo ou a caminhada.

No que se refere à sensação de segurança ao longo do trajeto foram registradas diversas reclamações: $62,4 \%$ das mulheres se mostraram insatisfeitas ou muito insatisfeitas, opinião também obtida em $53,5 \%$ das respostas masculinas. Além disso, a baixa iluminação e a reduzida quantidade de pessoas dentro da UFMT em alguns períodos do dia são algumas das causas para essa sensação de insegurança. Estudantes do período noturno reclamam sobre a falta de segurança dentro da universidade, visto que os guardas que trabalham no turno da noite possuem a função de proteção de patrimônio, portanto não realizam rondas e ficam situados em locais que não possuem visão das ruas da universidade.

O clima de Cuiabá pode desencorajar as pessoas que querem fazer suas viagens ativamente, - que é evidenciado pela avaliação de conforto, onde $54 \%$ dos respondentes estão insatisfeitos ou muito insatisfeitos. Basso e Corrêa (2014), em um estudo focado na cidade de Campo Grande (MS), discorrem que através da arborização adequada pode-se amenizar a sensação térmica no recinto urbano. Dessa forma, ao se pensar no incentivo ao uso dos modos ativos de transporte, o plantio de árvores no entorno e na própria instituição pode surtir efeitos positivos.

O desrespeito por parte dos usuários do transporte individual também é apontado como um problema pelos entrevistados, foram relatadas situações como: automóveis que avançam a faixa de pedestre, acelerar o veículo para induzir o cruzamento mais rápido das faixas, motociclistas realizando contornos e/ou utilizando calçadas e faixas de pedestre como faixa de rolamento. Quanto ao meio físico são observadas calçadas em mau estado de conservação, desniveladas, estreitas, e com sinalizações apagadas. A falta de ciclovias, tanto na UFMT quanto nos bairros do entorno, também são obstáculos constantemente repetidos nas respostas.

\section{CONCLUSÕES}

O caso da UFMT se demonstrou semelhante ao de muitas Universidades: o transporte individual é o principal modo de transporte utilizado para acessar o campus, sendo seguido 
pelo transporte público e o transporte ativo. Apesar desse cenário, o ambiente universitário favorece a criação e implementação de políticas voltadas à promoção da mobilidade sustentável. Dessa forma, antes de pensar em medidas e soluções, torna-se necessário compreender os padrões de deslocamento.

O presente artigo se propôs a determinar o perfil do usuário do transporte ativo dentro da UFMT e suas principais características. Sendo estes principalmente estudantes com faixa etária de 17 a 26 anos e renda abaixo de $R \$ 3.000$. Percebe-se que estes moram próximos à instituição, tendo como distância média de viagem $1,51 \mathrm{~km}$ e se deslocam a pé por, aproximadamente, 18 minutos e de bicicleta por 6 minutos.

Nas respostas abertas sobre os meios de transporte são observadas reclamações frequentes sobre: clima, problemas nas calçadas, iluminação, ausência de ciclovias ou ciclofaixas, e inexistência de paraciclos. Já a sensação de insegurança pode ser atribuída tanto à falta de policiamento quanto ao desrespeito das normas de trânsito por outros modos de transporte.

A partir dessa análise, é sugerida como pesquisa futura o levantamento de possíveis ações a fim de incentivar a mobilidade sustentável dentro do Campus e nos bairros no entorno, os quais têm maior potencial para uma mudança de postura dos viajantes.

\section{REFERÊNCIAS}

ANDRADE, M. O. DE; MEIRA, L. H.; MAIA, M. L. A. O transporte fretado para a viabilização da acessibilidade a um campus regional no interior do Nordeste. In: Anais do XXV ANPET -

Congresso de pesquisa e Ensino em Transportes, p. 1126-1137, 2011, Belo Horizonte- MG.

BALSAS, C. J. L. Sustainable transportation planning on college campuses. Transport Policy, v. 10, p. 35-49, 2003.

BASSO, J. M.; CORRÊA, R. Arborização urbana e qualificação da paisagem. Paisagem e Ambiente, n. 34, p. 129-148, 7 dez. 2014.

CARVALHO, G, S. D. de. Caracterização e análise da demanda por transporte em um campus universitário: o caso da UFRJ. 2016. 125 f. Dissertação (Mestrado) - Engenharia de Transportes, Programa de Engenharia de Transportes, COPPE/Universidade Federal do Rio de Janeiro, Rio de Janeiro, 2016.

DENATRAN. Manual de procedimentos para o tratamento de Pólos Geradores de Tráfego. 1. ed. Brasilia: DENATRAN/FGV, 2001.

KNEIB, E. C. Caracterização de empreendimentos geradores de viagens: Contribuição conceitual à análise de seus impactos no uso, ocupação e valorização do solo urbano. 2004. 168 f. Dissertação (Mestrado). Faculdade de Tecnologia, Departamento de Engenharia Civil e Ambiental, Universidade de Brasília, Brasília, 2004.

MIKIKI, F.; PAPADOPOULOU, P. Tackling mobility environmental impacts through the promotion of student active travel. Transportation Research Procedia, v. 24, p. 321-328, 2017.

MONTEIRO, F. B.; CAMPOS, V. B. G. Métodos de avaliação da qualidade dos espaços para ciclistas. In: Anais do XXV ANPET - Congresso de pesquisa e Ensino em Transportes, p. 12421253, 2011, Belo Horizonte- MG.

PARRA, M. C. Gerenciamento da mobilidade em campi universitários: problemas, dificuldades e possíveis soluções no caso da llha do Fundão. 2006. 120 f. Dissertação (Mestrado) - Engenharia de Transportes, Programa de Engenharia de Transportes, COPPE/Universidade Federal do Rio de Janeiro, Rio de Janeiro, 2006. 
PORTUGAL, L. DA S.; GOLDNER, L. G. Estudo de Pólos Geradores de Tráfego e de seus Impactos nos Sistemas Viários e de Transportes. 1. ed. São Paulo: Blucher, 2003.

RIGGS, W. Dealing with parking issues on an urban campus: The case of UC Berkeley. Case Studies on Transport Policy, v.2, p. 168-176, 2014.

SEGADILHA, A. B. P. Identificação dos fatores que influenciam na escolha da rota pelos ciclistas: Estudo de caso da cidade de São Carlos. 2014. 83 f. Dissertação (Mestrado) Engenharia Urbana, Programa de Pós-graduação em Engenharia Urbana, Universidade Federal de São Carlos, São Carlos, 2014.

STASKO, T. H.; BUCK, A. B.; GAO, H. O. Carsharing in a university setting: Impacts on vehicle ownership, parking demand, and mobility in Ithaca, NY. Transport Policy, v. 30, p. 262-268, 2013.

STEIN, P. P. Barreiras, motivações e estratégias para mobilidade sustentável no campus São Carlos da USP. 2013. 277 f. Dissertação (Mestrado) - Engenharia de Transportes, Programa de Pós-Graduação em Engenharia de Transportes, Escola de Engenharia de São Carlos da Universidade de São Paulo, São Carlos, 2013.

STEIN, P. P.; RODRIGUES DA SILVA, A. N. DA; JÚNIOR, C. A. P. DA S. Impactos nas distâncias de caminhada decorrentes de acessos exclusivos para pedestres em Campus Universitário.

Paranoá, v. 6, p. 113-121, 2012.

TOLLEY, T. Green campuses: cutting the environmental cost of commuting. Journal of Transport Geography, v. 4, n. 3, p 213-217, 1996.

UFMT. Anuário Estatístico 2018 - Ano base 2017. Disponível em:

<http://wwwl.ufmt.br/anuarioestatistico/arquivos/delfe92638319ba0be5960867481e363.pdf >. Acesso em: 25 mar. 2019. 